\title{
Quelques réflexions autour de la bourgeoisie québécoise, 1850-1914
}

\section{Paul-André Linteau}

Volume 30, numéro 1, juin 1976

URI : https://id.erudit.org/iderudit/303509ar

DOI : https://doi.org/10.7202/303509ar

Aller au sommaire du numéro

Éditeur(s)

Institut d'histoire de l'Amérique française

ISSN

0035-2357 (imprimé)

1492-1383 (numérique)

Découvrir la revue

Citer cette note

Linteau, P.-A. (1976). Quelques réflexions autour de la bourgeoisie québécoise, 1850-1914. Revue d'histoire de l'Amérique française, 30(1), 55-66.

https://doi.org/10.7202/303509ar d'utilisation que vous pouvez consulter en ligne.

https://apropos.erudit.org/fr/usagers/politique-dutilisation/ 


\section{QUELQUES RÉFLEXIONS AUTOUR DE LA BOURGEOISIE QUÉBÉCOISE, 1850-1914 ${ }^{1}$

\author{
PAUL-ANDRÉ LINTEAU \\ Département d'bistoire \\ Université du Québec à Montréal
}

Depuis quelques années, les préoccupations pour l'étude de la structure sociale prennent une part croissante dans les réflexions des historiens québécois. Les colloques et les congrès des dernières années, et les échanges qu'ils ont suscités, témoignent de cet intérêt.

S'inspirant de collègues français, britanniques ou américains, les historiens québécois se sont parfois lancés dans le domaine neuf et attrayant de l'histoire sociale. Leur volonté de découper les populations en les catégorisant et en les hiérarchisant s'est toutefois avérée une entreprise difficile. D'une part la réflexion théorique était déficiente. D'autre part, et surtout, le long sous-développement de la science historique au Québec faisait sentir ses effets: le nombre et le niveau des monographies existantes ne fournissaient guère de matière à une histoire globale.

Il fallait donc commencer plus modestement en étudiant des groupes ou des classes, dans des régions bien définies et pour des périodes limitées. En même temps que se poursuivait la réflexion théorique, il fallait accumuler des données suffisamment pertinentes. C'est la démarche qu'ont suivie depuis quelques années plusieurs historiens québécois et il faudra sans doute un certain temps de ce régime avant que l'on puisse comprendre avec rigueur et clarté

1 Ces réflexions ont eu comme point de départ mes recherches de doctorat; voir Paul-André Linteau, Histoire de la ville de Maisonneuve, 1883-1918, thèse de $\mathrm{Ph}$. D. (histoire) (Université de Montréal, 1975), 427 p., en particulier les chapitres 1, 2 et 7 . Elles se sont enrichies des discussions que j'ai eues avec de nombreux collègues de diverses universités; je les remercie collectivement. 
l'ensemble de la structure sociale québécoise du passé. D'ici là, il faut se contenter d'éléments qui restent fragmentaires. Les quelques réflexions qui suivent veulent être une contribution à cet effort collectif.

Mon intention n'est pas de régler le problème des classes sociales au Québec, mais d'en examiner une dimension. Plus précisément, mes propos concernent la bourgeoisie québécoise, pendant la période qui va du milieu du $\mathrm{XIX}^{\mathrm{e}}$ siècle à la Première guerre mondiale. Et encore m'en tiendrai-je à certains aspects de l'étude de cette classe.

Au premier abord cette bourgeoisie pouvait paraitre bien connue puisque, jusque-là, l'historiographie n'avait eu d'yeux que pour elle. Très tôt il fallut se rendre à l'évidence: si certains individus étaient connus, l'action et la composition de la bourgeoisie québécoise comme entité sociale ne l'étaient pas beaucoup.

Certes, un certain effort de systématisation a été entrepris depuis quelques années. Mais c'est une vision bipolaire des classes dirigeantes que l'on retrouve de façon plus ou moins nette chez des auteurs comme Marcel Rioux, Gilles Bourque et Nicole Frenette, Jean Hamelin. Il y a d'une part la haute bourgeoisie, propriétaire du capital et qui contrôle l'économie québécoise; elle est essentiellement anglophone même si parfois s'y glissent des Canadiens français, comme Sénécal ou Forget. Il y a d'autre part une petitebourgeoisie, formée surtout de petits commerçants, de membres des professions libérales et du clergé, dont les pouvoirs économiques sont restreints mais qui exerce un contrôle politique et idéologique sur la société québécoise; elle est essentiellement francophone.

Cette vision bipolaire s'avère rapidement insatisfaisante quand on entreprend des recherches plus poussées sur les milieux d'affaires québécois. Elle soulève en tout cas de sérieuses questions.

Que faire, par exemple, du grand nombre d'hommes d'affaires anglophones qui sont à la tête de petites et moyennes entreprises? $\mathrm{Si}$ on les classe parmi la haute bourgeoisie ne donne-t-on pas à ce concept une définition beaucoup trop élastique? Si on les rattache à la petite-bourgeoisie, que reste-t-il du caractère massivement francophone de celle-ci?

De même l'identification des francophones à la petite-bourgeoisie pose problème. Peut-on croire qu'une classe exerce un contrôle idéologique aussi poussé, et pendant aussi longtemps, sans que ce 
contrôle repose sur des bases matérielles? La participation des francophones au contrôle de l'activité économique est-elle aussi limitée qu'on le dit? Peut-on la restreindre aux rares individus - les Forget, les Sénécal, et quelques autres - qui sont généralement associés à la haute bourgeoisie? A-t-on suffisamment tenu compte de l'ensemble des secteurs d'activité, et en particulier du secteur foncier où les hommes d'affaires canadiens-français sont très actifs? A-t-on bien vu les nombreuses activités économiques qui se cachent derrière l'appellation «avocat» ou «notaire»?

Manifestement, il faut compléter ce cadre d'analyse. La période 1850-1914 est témoin d'une croissance économique importante au Québec qui amène une augmentation notable des effectifs de la bourgeoisie. Elle est aussi témoin des débuts d'un changement fondamental dans les structures du système capitaliste alors que s'amorce le passage du capitalisme de concurrence au capitalisme de monopole. Pour bien voir les effets de ces transformations sur la bourgeoisie au Québec il faut raffiner l'effort de systématisation et, en particulier, montrer les couches qui existent au sein de la bourgeoisie.

La bourgeoisie, définie par le critère commun de la propriété et de la possession du capital, n'en présente pas moins en son sein une hiérarchisation correspondant à des niveaux de pouvoir. Pour bien comprendre le rôle historique de la bourgeoisie au Québec, il importe d'identifier ceux de ses membres qui sont en mesure de déterminer les grandes orientations économiques et dont les décisions affectent l'ensemble du pays. C'est le niveau de contrôle des instruments de développement qui permettra d'effectuer la distinction. D'une part, ceux qui contrôlent les grandes institutions économiques et d'autre part ceux dont le rayon d'action est beaucoup moins étendu et qui n'exercent leur domination que sur des institutions de moyenne importance ou de portée régionale. La distinction n'est pas toujours facile à établir avec précision. Il y a, comme dans bien d'autres classifications, des cas-frontière, difficiles à trancher. Mais, ce qui nous intéresse ici, c'est d'identifier la tendance générale, de définir globalement les couches sans chercher à régler les cas individuels.

L'utilisation de ce critère de contrôle amène à considérer successivement la grande bourgeoisie, la moyenne bourgeoisie et la petite-bourgeoisie. 


\section{La grande bourgeoisie}

$\mathrm{Au}$ sommet se trouve la grande bourgeoisie que je définirais comme celle qui organise et contrôle le développement dans un contexte pancanadien et impérial. Elle dirige les institutions financières - banques, compagnies d'assurances et sociétés de fiducie - les plus importantes; les principales industries; les entreprises de chemin de fer et de navigation d'envergure continentale ou internationale; le grand commerce; les projets fonciers qui concernent d'immenses régions.

Les représentants de la grande bourgeoisie sont massivement concentrés à Montréal; on en trouve peu dans les autres villes du Québec car ils s'installent là où se trouve le principal centre de décision. Leurs effectifs sont peu nombreux et sont majoritairement d'origine anglo-écossaise bien que des bourgeois d'autres origines, dont quelques Canadiens français, réussissent à s'insérer dans le groupe. Les Smith, Stephen, Allan, Holt, Forget, Van Horne et Ross en sont les représentants les plus éminents.

Un rappel de la biographie de Donald Smith illustrera mon propos. Né en Écosse en 1820 , il commence sa carrière comme commis de la Compagnie de la Baie d'Hudson. Gravissant les échelons, il en devient Chief commissioner pour le Canada, en 1870, puis administrateur et gouverneur. Il est également un des membres fondateurs de la compagnie du Canadien Pacifique, en 1880. Il occupe en outre de hautes fonctions à la Banque de Montréal dont il est successivement vice-président (1882-1887), président (1887-1905) et président honoraire (1905-1914). Smith est donc à la fois un actionnaire important et un administrateur-clé de trois entreprises qui ont pesé d'un poids déterminant dans l'histoire économique du Canada. Il participe aussi à de nombreuses autres entreprises industrielles, commerciales et financières. Au cours de sa carrière, il amasse une immense fortune et, au tournant du siècle, il est devenu le plus riche bourgeois montréalais. Parallèlement à ces activités Donald Smith mène une fructueuse carrière politique; il est, pendant une vingtaine d'années, député au Parlement canadien, puis nommé, en 1897, à la Chambre des Lords de Grande-Bretagne. De 1896 jusqu'à sa mort, en 1914, il est Haut-Commissaire du Canada à Londres ${ }^{2}$.

2 Sur Donald Smith voir, entre autres, H.J. Morgan, Canadian Men and Women of the time: A Handbook of Canadian Biography (Toronto, William Briggs, 1898), 975-977. 
Smith et les autres qui œuvrent à ce niveau jouissent d'un pouvoir considérable et exercent un contrôle sur les grandes institutions qui marquent la société québécoise. Leur action ne se situe pas au même niveau que celle de la majorité des hommes d'affaires québécois; face à ces derniers ils occupent une position dominante. A mon avis, il y a lieu de réserver à ce groupe l'appellation de grande bourgeoisie.

Cette grande bourgeoisie est la principale responsable des orientations qui sont imprimées à l'économie canadienne entre 1850 et 1914, en particulier lors de la création de la Confédération et de la formulation de la National Policy. Elle ne présente pas toujours un front uni et ses effectifs se regroupent au sein de réseaux parfois antagonistes. Le plus important de ces réseaux de la grande bourgeoisie québécoise est formé par le groupe de la Banque de Montréal, du Canadien Pacifique et des sociétés qui leur sont associées. Smith, Stephen, Angus, Van Horne, Shaughnessey et Meredith en sont les porte-paroles les plus éminents. À l'aube du $\mathrm{XX}^{\mathrm{e}}$ siècle d'autres groupes émergent, en particulier celui du tandem HoltForget et de la Banque Royale. C'est l'époque des premières grandes fusions d'entreprises dans les secteurs bancaire, industriel et dans les services publics. Les travaux de Dales ${ }^{3}$, de Piédalue ${ }^{4}$, de Niosi ${ }^{5}$, montrent clairement le rôle moteur de la grande bourgeoisie montréalaise dans le passage au capitalisme de monopole.

Cette grande bourgeoisie québécoise a des liens avec les bourgeoisies de Grande-Bretagne et des États-Unis. Le Canada et le Québec étant des zones dépendantes au sein d'empires économiques, il serait illusoire de penser que la bourgeoisie québécoise puisse être parfaitement autonome. La fin de carrière de Smith en GrandeBretagne, où la pairie récompense son dévouement à l'Empire, et son association étroite avec la Compagnie de la Baie d'Hudson témoignent de la force de ces liens. Il en est de même de la participation de Van Horne à de nombreux projets américains de cons-

3 John H. Dales, Hydroelectricity and industrial development. Quebec, 18981940 (Cambridge, Harvard University Press, 1957), en particulier le chapitre 5.

4 Gilles Piédalue, Le Capital financier et l'industrie des pâtes et papiers au Québec (1920-1930), thèse de M.A. (histoire) (Université du Québec à Montréal, 1973), 166 p.; «Les groupes financiers et la guerre du papier au Canada, 19201930 », communication à la Société historique du Canada (1975); «Les groupes financiers au Canada (1900-1930), étude préliminaire», RHAF, 30, 1 (juin 1976): 3-34.

5 Jorge Niosi, "La Laurentide (1887-1928): pionnière du papier journal au Canada», RHAF, 29, 3 (décembre 1975) : 375-415. 
truction ferroviaire en Amérique latine. À l'opposé, il est difficile de souscrire à l'image simpliste d'une bourgeoisie canadienne ou québécoise qui ne serait qu'un valet des bourgeoisies impérialistes. Il y a de nombreuses oppositions entre elles. La résistance séculaire des grandes banques canadiennes à l'établissement de banques américaines au Canada en témoigne. De même que la violente guerre qui, dans les années 1920, au Québec, oppose le groupe de la Banque Royale et les intérêts Rockefeller dans le secteur des pâtes et papiers.

\section{La moyenne bourgeoisie}

À un niveau intermédiaire se situe une importante bourgeoisie moyenne que les historiens ont tendance à négliger. Elle vise à organiser et à contrôler le développement dans un contexte régional. Elle oriente ses activités vers les institutions financières d'envergure régionale, la petite et moyenne entreprise commerciale ou industrielle, la promotion urbaine et la construction des chemins de fer dits de colonisation qui sont en réalité des voies de communication régionales.

Ses représentants sont beaucoup plus nombreux que ceux de la grande bourgeoisie. Ils sont répartis différemment sur le territoire puisqu'on les retrouve dans les principales villes du Québec.

Le cas d'Alphonse Desjardins illustrera cette fois mon propos. Desjardins, qu'il ne faut pas confondre avec son homonyme fondateur des Caisses populaires, est né en 1841 et mort en 1912. Il est particulièrement actif dans le secteur financier. Président de la Banque Jacques-Cartier de 1880 jusqu'à la fermeture de l'institution en 1899, il en est pendant plusieurs années le principal actionnaire. Il siège aussi au conseil d'administration de sociétés de crédit foncier et d'assurances. Son action ne se limite pas au secteur financier. On le retrouve dans le domaine industriel - il fonde une entreprise de fabrication de briques - et dans le secteur foncier - il est promoteur urbain à Maisonneuve, une ville de la banlieue montréalaise. Avocat de formation et, pendant un certain temps, directeur du journal Le Nouveau Monde, il est un des leaders du groupe ultramontain de Montréal. Député conservateur au Parlement fédéral de 1878 à 1892, puis sénateur de 1892 à 1896, il est ministre sous Bowell et Tupper en 1896. Il avait été aussi maire de Montréal en 1893-1894. La défaite du parti conservateur fédéral en 1896 met fin à sa carrière politique. L'élection de Desjardins à la présidence de la Cham- 
bre de commerce du district de Montréal, en 1899, témoigne du prestige dont il jouit dans les milieux d'affaires ${ }^{6}$.

Les biographies d'Alphonse Desjardins et de Donald Smith présentent de nombreux points de comparaison. Tous deux mènent parallèlement une carrière politique et une carrière dans les affaires. Leur activité économique est diversifiée et dans les deux cas on les retrouve simultanément dans les secteurs financier, industriel et foncier. De toute évidence, nous avons affaire à deux bourgeois, caractérisés par le critère commun de la propriété du capital. Mais audelà de cela, il est aussi évident que le degré de contrôle qu'ils exercent sur l'économie québécoise ou canadienne n'est pas le même. Prenons simplement l'exemple de leur association au capital bancaire. Smith et Desjardins sont pendant près d'une vingtaine d'années présidents de banque, l'un à la Banque de Montréal, l'autre à la Banque Jacques-Cartier. Là s'arrête la similitude. La Banque de Montréal a, par exemple, en juillet 1896 , un actif de $\$ 59,289,254$; elle est agent financier du gouvernement du Québec et de celui du Canada; elle étend son emprise sur l'ensemble du territoire canadien. La Banque Jacques-Cartier, de son côté, a un actif vingt fois moindre $(\$ 3,040,274)$; ses opérations sont surtout concentrées dans la région de Montréal. Le degré de pouvoir et de contrôle des deux institutions et de leurs présidents n'est pas comparable. Ce n'est pas un hasard que Desjardins soit nommé au Sénat, alors que Smith termine sa carrière à la Chambre des Lords.

Tenter, dans l'état actuel des recherches, d'établir les effectifs de la moyenne bourgeoisie québécoise serait d'ailleurs illusoire. Ceux-ci sont certainement beaucoup plus considérables que ceux de la grande bourgeoisie et l'expansion économique que connaît le Québec entre 1850 et 1914 provoque l'accroissement de leur nombre. Un simple coup d'œil aux répertoires d'affaires, pour ces deux dates, suffit pour s'en convaincre.

S'ils sont tenus à l'écart des très grandes institutions financières, les représentants de la moyenne bourgeoisie sont quand même présents dans tous les secteurs de l'activité économique. Ils se dotent

6 Sur Alphonse Desjardins voir: Le Devoir, 5 juin 1912, 6; H. J. Morgan, Canadian Men and Women of the time (Toronto, 1912), 320; J.-C. Lamothe, Histoire de la corporation de la cité de Montréal (Montréal, Montreal Printing \& Publishing Co., 1903), 315-317; "Liste des actionnaires des banques autorisées du Canada... ", Documents de la session, 1874-1900; P.-A. Linteau, Histoire de la ville de Maisonneuve, 180-182, 201-207. 
d'institutions à leur mesure comme les banques régionales. Jusqu'à la fin du XIX ${ }^{\mathrm{e}}$ siècle ils sont massivement présents au sein des institutions politiques, aux trois paliers de gouvernement, et plus spécifiquement au niveau municipal où les décisions affectent leurs intérêts économiques immédiats.

Le prototype de la moyenne bourgeoisie québécoise est la bourgeoisie de la capitale. La ville de Québec a perdu, après 1850, sa participation active aux grandes institutions économiques pancanadiennes et elle connaît même, pendant les dernières décennies du siècle, une période de relative stagnation. La solution, pour la bourgeoisie de Québec, réside dans la définition d'une véritable économie régionale qui passe non seulement par l'intégration des zones rurales environnantes mais aussi par la création d'un vaste hinterland, en annexant les zones périphériques. Ce qui explique le lancement, par la bourgeoisie de Québec, de trois grands projets ferroviaires: le chemin de fer de la rive Nord avec l'objectif irréaliste de ravir à Montréal le commerce pancanadien, mais qui permettra tout de même une meilleure intégration de la zone intermédiaire de Québec à Trois-Rivières; le chemin de fer Lévis et Kennebec (plus tard Québec Central) qui vise à placer dans l'orbite de la capitale la Beauce et une partie des Cantons de l'est tout en resserrant les liens entre Québec et Sherbrooke; enfin le chemin de fer Québec et LacSaint-Jean qui assurera l'annexion du vaste territoire saguenayéen. Dans ce dernier cas, Normand Séguin a bien montré comment le chemin de fer a été un instrument majeur de pénétration du capital de Québec au Lac-Saint-Jean ${ }^{7}$. Les marchands de bois, les commerçants en gros, les sociétés de prêt hypothécaire représentent autant de facettes des activités de la moyenne bourgeoisie de la capitale. Celle-ci se dote d'instruments qui lui sont propres, comme la Banque Nationale ou la Banque de Québec. Les Beaudet, Shehyn, Chinic, Thibaudeau, illustrent, parmi d'autres, la diversité des activités de ce groupe.

La présence de banques régionales est à mettre en relation avec l'existence d'une moyenne bourgeoisie. Pensons à la Eastern Townships Bank à Sherbrooke, ainsi qu'à la Banque de St-Hyacinthe et à la Banque de Saint-Jean. Cependant la moyenne bourgeoisie québécoise est surtout présente à Montréal. Les Rodier, Rolland,

7 Normand Séguin, Notre-Dame d'Hébertville, 1850-1900, une paroisse de colonisation au XIXe siècle, thèse de Ph. D. (histoire) (Université d'Ottawa, 1976), $356 \mathrm{p}$. 
Beaubien, Beaudry, Rivard, Desjardins, Préfontaine, Viau, Cherrier, Béique, Paré, Barsalou, Valois, Coursol, Delisle, Letourneux en sont des représentants bien connus, mais il y en a de nombreux autres qu'il importerait de mieux identifier. Elle aussi se dote d'institutions financières comme la Banque d'Hochelaga ou la Banque JacquesCartier.

La composition ethnique de la moyenne bourgeoisie parait être plus diversifiée que celle de la grande bourgeoisie. Certes, on y trouve un fort contingent d'Anglais et d'Écossais. On doit s'attendre à y trouver aussi un certain nombre d'Irlandais. Mais ce qui me paraît encore plus significatif est la présence, beaucoup plus considérable qu'on ne l'a cru jusqu'ici, quoique difficile à évaluer, des Canadiens français. Dans ce dernier cas il me semble y avoir eu sous-évaluation de la part des historiens. Comment l'expliquer? Certainement par la relative jeunesse des études d'histoire économique sur le Québec contemporain. Pendant près de deux décennies il n'y avait guère qu'Albert Faucher qui s'évertuait à prêcher dans le désert. Les informations sur les faits économiques du Québec au tournant du siècle commencent à peine à être mises à jour. Les monographies sur des entreprises de dimension moyenne sont à peu près inexistantes. Tout le secteur foncier, dans lequel les bourgeois francophones se sont manifestés, a été ignoré par les historiens. On a allègrement classé parmi la petite-bourgeoisie les avocats en oubliant que souvent cette profession n'était qu'une dimension de leur activité et que le phénomène nord-américain de l'avocat-brasseur d'affaires s'était aussi manifesté au Québec. Alphonse Desjardins dont nous avons parlé plus haut et que les ouvrages décrivent souvent comme «avocat et journaliste» en est un bel exemple. Les recherches les plus récentes m'incitent à croire qu'il faut réévaluer la participation des effectifs francophones au sein de la bourgeoisie en tenant compte d'une présence significative au niveau intermédiaire.

Voilà donc précisées les caractéristiques de la moyenne bourgeoisie québécoise. Comment évolue-t-elle entre 1850 et 1914? Il faut dire qu'elle est identifiée aux secteurs de l'économie qui en sont encore à l'étape concurrentielle. Le capitalisme de concurrence étant la forme dominante pendant la seconde moitié du XIX ${ }^{\mathrm{e}}$ siècle, la moyenne bourgeoisie se trouve dans une conjoncture favorable. Il n'en est plus de même à partir du début du $\mathrm{XX}^{\mathrm{e}}$ siècle. La monopolisation entraîne une concentration croissante des pouvoirs économiques et du contrôle entre les mains de la grande bourgeoisie. La moyenne bourgeoisie est peu à peu évincée de plusieurs secteurs 
et elle perd les instruments autonomes qu'elle s'était donnés pendant la période précédente. Cela est particulièrement évident dans le secteur bancaire où les géants absorbent la plupart des institutions régionales. Il $\mathrm{y}$ a là une transformation structurelle qui n'élimine pas la moyenne bourgeoisie, mais qui diminue son emprise et qui accroît l'écart entre elle et la grande bourgeoisie. À l'aube de la Première guerre mondiale, les rapports de force sont déjà changés.

Entre ces deux couches, la différence n'en est donc pas une de nature mais de degré. Il s'agit d'une même bourgeoisie, possédant le capital, et dominant l'activité économique, au sein de laquelle se manifestent des contradictions, des luttes d'intérêt et où se dégagent des niveaux de pouvoir.

\section{La petite-bourgeoisie}

Pour certains, la petite-bourgeoisie constitue la couche inférieure de la bourgeoisie. Pour les auteurs marxistes, cependant, elle ne fait pas partie de la classe bourgeoise, car, quoique possédant une partie du capital, elle n'exploite que peu ou pas le travail salarié. Mais qu'elle en fasse partie ou non, la petite-bourgeoisie québécoise de la période 1850-1914 participe au contrôle de l'activité économique et est souvent associée à certains éléments de la bourgeoisie.

Évidemment, elle n'obtient qu'une part restreinte du contrôle de l'économie, même si ses effectifs sont plus nombreux. Son action s'exerce au niveau local - le village, la paroisse, le quartier. Ses secteurs d'intervention privilégiés sont le commerce de détail et les opérations foncières. On associe généralement à la petite-bourgeoisie un groupe - les membres des professions libérales - qui participe souvent aux activités économiques de celle-ci ou qui, à tout le moins, lui apporte son support. L'ensemble formant ce groupe de notables qui, dans une petite municipalité ou une paroisse, contrôle le conseil municipal, la commission scolaire et la fabrique.

La composition ethnique de ce groupe devrait être semblable à celle de la population qu'elle dessert, c'est-à-dire majoritairement francophone. Il ne serait pas surprenant toutefois de trouver chez les petits commerçants une surreprésentation d'immigrants. Cela resterait cependant à vérifier.

La position de la petite-bourgeoisie face à l'ensemble de la bourgeoisie est, elle aussi, marquée par les transformations écono- 
miques de la période 1850-1914. L'isolement qui prévaut dans plusieurs régions du Québec au milieu du XIX ${ }^{\mathrm{e}}$ siècle favorise le contrôle de l'économie locale par cette petite-bourgeoisie. Toutefois l'amélioration des communications favorise la mainmise croissante de la bourgeoisie des centres régionaux sur l'économie locale. La petite-bourgeoisie ne disparaît pas pour autant mais elle est de plus en plus inféodée à la moyenne bourgeoisie; elle devient l'intermédiaire, l'agent local au service de celle-ci. Des rapports de dépendance étroits s'établissent entre les deux; ils se manifestent également aux plans politiques et idéologiques.

C'est donc le niveau de contrôle qui définit et caractérise ces trois couches et non pas le revenu ou la fortune. En outre cette typologie montre la nécessité, pour comprendre la structure sociale, de privilégier des critères socio-économiques plutôt que des critères ethniques.

Est-ce à dire que l'origine ethnique n'entre pas en ligne de compte dans la formation de la structure sociale? Rien ne semble moins sûr. Par exemple, l'ostracisme de la haute bourgeoisie angloécossaise de Montréal à l'endroit des Juifs de la métropole est trop connu pour être passé sous silence. En serait-il de même face aux Irlandais et aux Canadiens français?

Sans être un critère premier, l'ethnie est tout de même un facteur d'accélération ou de freinage. L'accession à la grande et moyenne bourgeoisie se fait généralement par l'intermédiaire de groupes qui contrôlent une ou plusieurs institutions et ont accès à des réseaux d'information et à des sources de capital. Le recrutement au sein de ces groupes se fait souvent à partir de facteurs comme: le lien de parenté, les relations d'études, etc., qui d'une certaine façon se retrouvent dans le critère ethnique. Il est plus difficile de pénétrer dans les groupes - peu nombreux - qui forment la haute bourgeoisie que dans ceux qui composent la moyenne bourgeoisie.

Dans ce contexte se pose le problème de la marginalisation de la bourgeoisie canadienne-française après la Première guerre mondiale $^{8}$. Ce phénomène s'explique d'abord et avant tout par une trans-

8 Jusqu'ici ce phénomène a surtout été expliqué par des facteurs culturels et politiques. Voir Paul-André Linteau et René Durocher, dir., "Le «retard» du Québec et l'infériorité économique des Canadiens français (Montréal, Boréal Express, 1971), $127 \mathrm{p}$. 
formation économique: la tendance à la monopolisation. Ce ne sont pas les Canadiens français qui sont marginalisés, mais l'ensemble de la moyenne bourgeoisie au sein de laquelle se trouvent de nombreux francophones. La monopolisation est faite par et pour la grande bourgeoisie qui agit de plus en plus au niveau pancanadien et international. Les hommes d'affaires canadiens-français, dont les bases économiques sont régionales, ne peuvent guère espérer jouer un rôle important à ce niveau.

Ce sont là quelques réflexions qui, je l'espère, favoriseront les échanges de vue et stimuleront les recherches. Il m'apparaît en particulier important de préciser la participation de la bourgeoisie francophone au développement économique du Québec entre 1850 et 1914. Pour cela il faut identifier les principaux groupes financiers, étudier l'évolution des banques et des autres entreprises importantes à propriété francophone. Les recherches en cours permettent d'espérer que cette dimension de l'histoire québécoise sera bientôt mieux connue. 